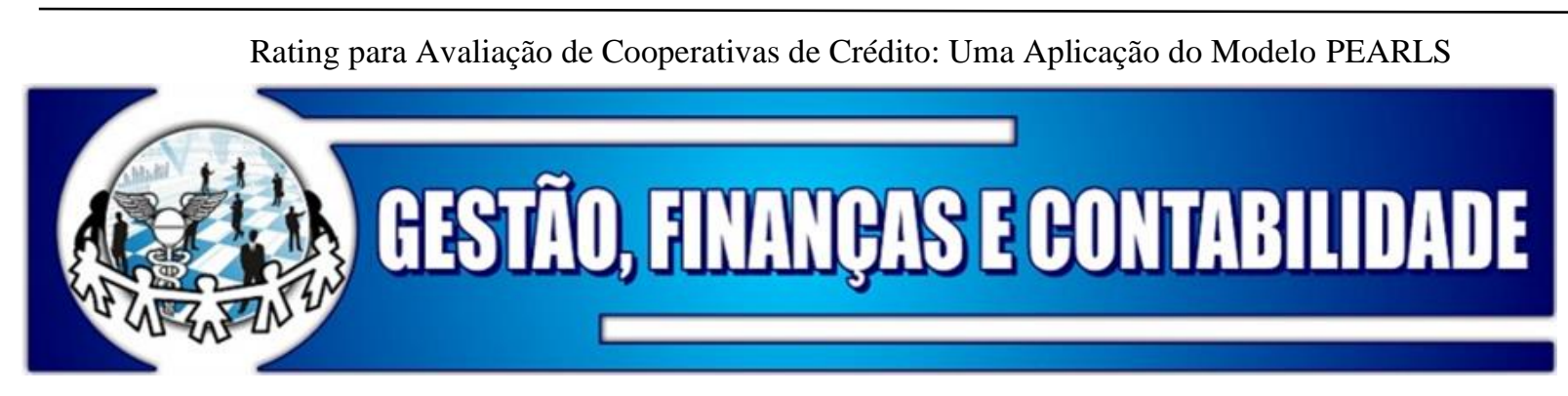

\title{
RATING PARA AVALIAÇÃO DE COOPERATIVAS DE CRÉDITO: UMA APLICAÇÃO DO MODELO PEARLS
}

\section{RATING FOR ASSESSMENT OF CREDIT UNIONS: AN APPLICATION OF THE PEARLS MODEL}

\section{RATING PARA LA EVALUACIÓN DE COOPERATIVAS DE CRÉDITO: UNA APLICACIÓN DEL MODELO PEARLS}

\author{
Paulo Fabrício Huscher \\ https://orcid.org/0000-0001-9160-4827 \\ Gerente do Banco do Brasil \\ Mestre em Gestão de Cooperativas pela Pontifícia Universidade Católica do Paraná \\ E-mail: pfh251@gmail.com
}

Vilmar Rodrigues Moreira

https://orcid.org/0000-0003-1796-6752

Professor do Programa de Pós-graduação em Gestão de Cooperativas (PPGCOOP/PUCPR)

Doutor em Administração pela Fundação Getúlio Vargas (EAESP/FGV)

E-mail: vilmar.moreira@ pucpr.br

Rodrigo Alves Silva

https://orcid.org/0000-0002-4539-2568

Professor da Universidade Tecnológica Federal do Paraná (UTFPR)

Doutor em Administração (FEAC/USP)

E-mail: alvesr@utfpr.edu.br

\section{RESUMO}

Para executar suas atividades as cooperativas de crédito, assim como outras organizações financeiras, realizam operações de empréstimo e intermediação, oferecendo risco de crédito aos demais agentes. Este artigo tem por objetivo apresentar um modelo de rating para avaliação de cooperativas de crédito. A pesquisa utilizou dados financeiros disponibilizados pelo sistema COSIF do Banco Central do Brasil, o modelo PEARLS de análise econômico-financeira de cooperativas de crédito e a metodologia de classificação do Fundo Garantidor (FGCOOP). O modelo de rating foi estimado por meio de regressão logística multinomial. A acurácia total foi de $80,1 \%$. Com os resultados obtidos, considerando o grau de acurácia do modelo, é possível verificar que o uso do modelo PEARLS permitiu o desenvolvimento de um modelo para monitoramento do risco de crédito que as cooperativas representam para o mercado financeiro. O modelo se mostrou simples e com relevância prática para os agentes. A relevância acadêmica está relacionada à metodologia de avaliação econômico-financeira de cooperativas de crédito, podendo ser usada como referência para trabalhos futuros. Este estudo também se justifica pela incipiência de pesquisas sobre ratings para avaliação de instituições financeiras no Brasil.

Palavras-chave: Risco de Crédito. Rating de Crédito. Cooperativas de Crédito. Modelo PEARLS. 


\begin{abstract}
As other financial organizations, to perform their activities credit unions carry out loans and intermediation operations, offering credit risk to other agents. The goal of this paper is to present a rating model for assessment of credit unions. It was used financial data available through the COSIF system of Central Bank of Brazil, the PEARLS model of economic-financial assessment and the classification methodology of the Brazilian Guarantee Fund for Credit Unions (FGCOOP). The rating model was estimated through multinomial logistic regression. The total accuracy was $80.1 \%$. With the model results and considering its high accuracy degree, it is possible to verify that the use of PEARLS model allowed the development of a model for monitoring the risks that credit unions represent to financial market. The achieved rating model is simple and has a practical relevance to agents. The academic relevance is related to the methodology for economic-financial assessment of credit unions which can be a reference for future studies. Moreover, the relevance is highlighted due to lack of studies on ratings to assess financial firms in Brazil.
\end{abstract}

Keywords: Credit Risk. Credit Rating. Credit Unions. PEARLS Model.

\title{
RESUMEN
}

Para el desarrollo de sus actividades, las cooperativas de ahorro y crédito, así como otras entidades financieras, realizan operaciones de préstamo e intermediación, ofreciendo riesgo crediticio a otros agentes. Este artículo tiene como objetivo presentar un modelo de calificación para evaluar las cooperativas de ahorro y crédito. La investigación utilizó datos financieros puestos a disposición por el sistema COSIF del Banco Central de Brasil, el modelo PEARLS de análisis económico y financiero de las cooperativas de ahorro y crédito y la metodología de clasificación del Fondo de Garantía (FGCOOP). El modelo de calificación se estimó mediante regresión logística multinomial. La precisión total fue del 80,1\%. Con los resultados obtenidos, considerando el grado de precisión del modelo, es posible verificar que el uso del modelo PEARLS permitió desarrollar un modelo de seguimiento del riesgo que las cooperativas de ahorro y crédito representan para el mercado financiero. El modelo resultó ser simple y con relevancia práctica para los agentes. La relevancia académica está relacionada con la metodología de evaluación económica y financiera de las cooperativas de ahorro y crédito, que puede ser utilizada como referencia para trabajos futuros. Este estudio también se justifica por la falta de investigación sobre calificaciones para la evaluación de instituciones financieras en Brasil.

Palabras clave: Riesgo de crédito. Calificación crediticia. Cooperativas de crédito. Modelo PEARLS.

\section{INTRODUÇÃO}

O mercado financeiro é o setor da economia que é responsável por captar a poupança da sociedade e canalizá-la para as diversas atividades - agricultura, indústria, comércio e serviços. Em um extremo estão os investidores que proveem os recursos (poupanças) e no outro, os tomadores de capital. Esses aplicam os recursos nas áreas em que atuam e, com o rendimento, pagam os investidores. Dessa forma o mercado financeiro tem papel importante no desenvolvimento da produção, na distribuição e no consumo de bens e serviços nas economias capitalistas. Sob o ponto de vista econômico-financeiro, o mercado financeiro é o local onde se opera os excedentes financeiros dos credores convertido em créditos compatíveis com as necessidades dos tomadores. Para que o mercado possa se desenvolver de forma sustentável e os excedentes sejam intercambiados de maneira satisfatória e eficaz, é necessária uma gestão racional que garanta confiabilidade, segurança e previsibilidade. 
O sistema financeiro é um conjunto de instituições que se dedicam a propiciar condições satisfatórias para a manutenção de um fluxo de recursos entre poupadores e investidores. Redes de instituições financeiras públicas e privadas, sejam bancos ou cooperativas de crédito, intermediam essas operações e fazem funcionar o sistema. Assim, um dos focos principais de qualquer instituição financeira é em emprestar dinheiro. O que implica, consequentemente, por assumir riscos de crédito.

Crédito é a entrega de um valor presente mediante a promessa de pagamento futuro. $\mathrm{O}$ risco de crédito é o evento relacionado ao não recebimento desse crédito no prazo combinado. A ocorrência desse evento, que é incerto, gera risco de perda financeira decorrente da incapacidade da contraparte de uma operação de honrar seus compromissos. Assim, o risco de crédito envolve três elementos: o evento relacionado à inadimplência (não pagamento), a chance desse evento ocorrer (probabilidade) e o impacto financeiro (perdas).

O monitoramento do risco de crédito é uma atividade importante na área de Finanças Corporativas. As preocupações com esse tipo de risco cresceram nos anos de 1990 por conta das dramáticas alterações econômicas, políticas e tecnológicas em todo o mundo. Nas últimas duas décadas tais preocupações se intensificaram, em especial no que tange à capacidade dos bancos em monitorar o risco de suas operações. Isso ficou mais evidente desde a crise econômica conhecida como crise do subprime, que contagiou os mercados em 2008 (NAKAMURA; ROSZBACH, 2018). Essa crise foi caracterizada pelo não cumprimento dos contratos hipotecários classificados como "Triple $A$ " nos EUA, que favoreceu a deterioração nos balanços intermediários afetando o fluxo de fundos entre credores e devedores (CREPALDI; PAYÉS, 2015).

Ao longo do tempo os bancos têm evoluído seus sistemas de captação e processamento de informações de forma a oferecer mecanismos melhorados de previsão e monitoramento dos riscos de crédito (NAKAMURA; ROSZBACH, 2018). Essa disponibilidade de informações tem permitido o desenvolvimento de modelos que utilizam algoritmos simples e que produzem graus aceitáveis de previsão de solvência. Essas ferramentas são importantes para maximizar o acesso à informação e fomentar o mercado financeiro de crédito como um todo (BALIOS; THOMADAKIS; TSIPOURI, 2016).

Os modelos internos de rating são exemplos de ferramentas de análise de risco de crédito. Esses modelos são baseados em pontuação e classificam os tomadores num conjunto de classes previamente definidas. Tais classes são categorias ordinais em função do nível de risco que o tomador oferece ao emprestador. Assim, o rating de crédito, ou classificação de risco de crédito, é um instrumento cujo objetivo é atribuir um grau que sintetiza o risco do não cumprimento das obrigações de crédito por parte do tomador.

No Sistema Financeiro Nacional (SFN) brasileiro as cooperativas de crédito representam pouco menos de 5\% do mercado financeiro. No entanto, essas instituições financeiras vêm apresentando um crescimento consistente ao longo do tempo. Apenas em 2017 as cooperativas aumentaram em $8 \%$ o número de cooperados, em $15 \%$ sua carteira de crédito e em 16\% a sua captação, segundo o Panorama do Cooperativismo de Crédito do Banco Central do Brasil - BACEN (2017).

Como a operação das cooperativas de crédito está integrada ao SFN, essas instituições captam recursos no mercado e provêm liquidez aos seus títulos junto a bancos e outros agentes financeiros. As cooperativas captam recursos através de depósitos, podendo ser através de títulos a prazo (remunerados), saldo em conta corrente (depósito à vista) e por meio da cota capital dos associados. Barroso e Bialoskorski Neto (2012) citam também a captação através de recursos de repasse, normalmente de outras instituições financeiras. Os autores ressaltam que não há impedimento de as cooperativas transacionarem recursos com outras instituições, desde que estas operações tragam benefícios aos seus cooperados. Assim, é de grande importância o monitoramento do risco financeiro das cooperativas, uma vez que elas 
representam risco de crédito para outros agentes.

Em suma, estudos sobre o risco de crédito que as cooperativas de crédito representam se fundamentam em três razões: (i) as cooperativas podem se tornar inadimplentes; (ii) o crescimento da participação e importância das cooperativas de crédito no SFN nos últimos anos; (iii) o dinamismo do mercado financeiro nacional.

Este estudo também se justifica pela incipiência de pesquisas sobre ratings para avaliação de instituições financeiras no Brasil. Segundo Lima, Fonseca, Silveira e Assaf Neto (2018), o mercado financeiro brasileiro apresenta um quadro institucional e competitivo peculiar devido, sobretudo, ao seu processo de reestruturação e consolidação observado a partir dos anos 1990. Segundo os autores, é oportuno um melhor entendimento dos determinantes dos ratings das instituições financeiras atuantes no mercado financeiro. Este artigo contribui para o preenchimento dessa lacuna tendo como foco o sistema cooperativo de crédito.

Este estudo considerou o risco que as cooperativas de crédito representam para um grande banco de varejo nacional, denominado aqui como Banco X. Verificou-se que existe uma necessidade de aperfeiçoamento da metodologia atual de análise do risco de crédito, que carece de uma revisão criteriosa com vistas a melhorar o grau de acurácia, confiabilidade e modernidade da técnica originalmente adotada para sua construção. O Banco X é um dos principais bancos do país. De acordo com critério de ativos totais, que mede o tamanho das instituições financeiras no Brasil, o Banco X encontra-se entre os cinco maiores.

Este artigo aborda o risco de crédito sob o ponto de vista de instituições financeiras que transacionam crédito com cooperativas de crédito. O objetivo foi ajustar um modelo de rating para a classificação do risco de crédito que as cooperativas representam para os agentes econômicos, incluindo o Banco X.

\section{FUNDAMENTAÇÃO TEÓRICA}

\subsection{INSTITUIÇÕES FINANCEIRAS: COOPERATIVAS DE CRÉDITO E BANCOS MÚLTIPLOS}

As cooperativas são sociedades de pessoas, não de capital. Essa é uma das principais diferenças entre as sociedades cooperativas e a maioria das outras sociedades, como os bancos, as chamadas empresas de capital. Nestas últimas cada ação vale um voto. Nas cooperativas, cada membro vale um voto. Devido a multiplicidade de funções e atribuições que as sociedades cooperativas apresentam, estas assumem de forma concomitante o papel de fiscalizadoras, administradoras, usuárias e clientes, bem como de fornecedores de serviços e de recursos para os seus cooperados (BIALOSKORSKI NETO, 2012).

Com base em uma personalidade jurídica própria, as cooperativas de crédito são instituições financeiras que se mostram como privadas, com a finalidade de prestar serviços financeiros que implicam captar recursos e os repassar com juros mais baixos aos seus cooperados. Tais instituições pertencem ao Sistema Financeiro Nacional e têm como órgão regulador o Banco Central do Brasil, que além de fiscalizar as que estão em funcionamento, é o órgão que emite autorização para a criação de novas cooperativas de crédito. De acordo com sua atuação e a áreas de desenvolvimento de seus negócios, as cooperativas são denominadas (MEINEN, 2014):

- $\quad$ Cooperativas Singulares, de $1^{\circ}$ grau: constituídas por número mínimo de vinte pessoas físicas para prestação de serviços aos cooperados ou para a produção de bens e serviços em conjunto. Excepcionalmente, podem admitir pessoas jurídicas que tenham por objetivo as mesmas ou correlatas atividades das pessoas físicas associadas. Podem atuar em localidades distintas à da sede (considerando sua área de atuação definida em seu estatuto), por meio de Pontos de Atendimento Cooperativo - PAC.

- $\quad$ Cooperativas Centrais ou Federações, de $2^{\circ}$ grau: constituídas de no mínimo três 
cooperativas singulares, têm como objetivo a prestação de serviços às singulares e/ou outros associados, no sentido de organizar, em maior escala, as atividades econômicas. Excepcionalmente, pode admitir cooperados individuais que se dedicam às mesmas atividades das singulares e seus associados.

- Confederações de Cooperativas, de $3^{\circ}$ grau: organização das federações ou mesmo cooperativas centrais em busca de coordenar e orientar atividades que se vinculam com as filiadas.

A denominação sistema cooperativo é utilizado quando as cooperativas de crédito estão organizadas em mais de um grau. Algumas das cooperativas de crédito estão organizadas nos três graus, formando um sistema, que contam também com bancos cooperativos.

Os bancos múltiplos por sua vez são instituições consideradas intermediárias, que apresentam as funções de captação, crédito e pagamento. A captação de recursos de investidores por meio de depósitos é remunerada por uma taxa de captação. Posteriormente, os recursos são aplicados por meio de crédito (empréstimos) a terceiros (devedores). Os devedores pagam uma taxa de juros por esses empréstimos. Um dos ganhos dos bancos está no chamado spread bancário, que significa a diferença entre a taxa de captação paga aos investidores e a taxa de juros paga pelos tomadores de crédito (SILVA, 2013). crédito.

No Quadro 1 são apresentadas as principais diferenças entre bancos e cooperativas de

Quadro 1 - Diferenças entre cooperativas de crédito e bancos

\begin{tabular}{l|l}
\hline \multicolumn{1}{c}{ Cooperativas de crédito } & Bancos \\
\hline São sociedades de pessoas & São sociedades de capital
\end{tabular}

$\mathrm{O}$ voto tem peso igual para todos (uma pessoa, um voto)

As decisões são partilhadas entre muitos

Os administradores-líderes são do meio (associados)

O usuário é o próprio dono (cooperado)

Toda a política operacional é decidida pelos próprios usuários/donos (associados)

Não podem distinguir: o que vale para um, vale para todos (art. da Lei $\mathrm{n}^{\circ}$ 5.764/71)

Não discriminam, servindo a todos os públicos

Não restringem, tendo forte atuação nas comunidades mais remotas

A atividade mercantil não é cogitada (art. 79, parágrafo único, da Lei $\mathrm{n}^{\circ}$ 5.764/71

O preço das operações e dos serviços tem como referência os custos e como parâmetro as necessidades de reinvestimento

O relacionamento é personalizado/individual, com o apoio da informática

Estão comprometidas com as comunidades e os usuários

Desenvolvem-se pela cooperação

O lucro está fora do seu objeto, seja pela natureza, seja por determinação legal (art. $3^{\circ}$ da Lei no 5.764/71)
O poder é exercido na proporção do número de ações

As deliberações são concentradas

Os administradores são terceiros (homens de mercado)

O usuário das operações é cliente

O usuário não exerce qualquer influência na definição dos produtos e na sua precificação

Podem tratar distintamente cada usuário

Preferem o público de maior renda e as maiores corporações

Priorizam os grandes centros (embora não tenham limitação geográfica)

Têm propósitos mercantilistas

A remuneração das operações e dos serviços não tem parâmetro/limite

Atendem em massa, priorizando, ademais o autosserviço

Não têm vínculo com a comunidade e o públicoalvo

Avançam pela competição

Visam ao lucro por excelência 
Huscher, Moreira e Silva (2020)

Rating para Avaliação de Cooperativas de Crédito: Uma Aplicação do Modelo PEARLS

O excedente (sobras) é distribuído entre todos (usuários), na proporção das operações individuais, reduzindo ainda mais o preço final pago pelos cooperados e aumentando a remuneração de seus investimentos
O resultado é de poucos donos (nada é dividido com os clientes

Fonte: Meinen (2014)

\subsection{RATING DE CRÉDITO}

$\mathrm{O}$ rating é uma classificação de risco em forma de nota que se atribui a um emissor de dívida. Essa nota propicia a análise de crédito conforme a avaliação acerca de capacidade e disposição em honrar de forma pontual a dívida contraída.

Os ratings de crédito são vistos pelos investidores como uma forma de indicação da probabilidade de recebimento do capital que foi aplicado, conforme acordo realizado na época da realização do investimento. Assim, o rating se mostra como instrumento importante para o mercado ao propiciar dados potenciais de credores em uma opinião independente sobre o risco de crédito que se vincula com a dívida do emissor em análise (SILVEIRA; TAKAMATSU; AVELINO, 2017). Os ratings de crédito podem ser aplicados em diversas formas de emissões e não se limitam a países, estados ou municípios. Também são aplicados a instituições financeiras e seguradoras que aplicam operações estruturadas e garantidas por recebíveis e ativos financeiros.

O estabelecimento de ratings de países, estados, municípios e grandes corporações é feito por agências especializadas. Essas agências especializam-se na avaliação da capacidade creditícia de emitentes de títulos corporativos e públicos. Seu trabalho é informar aos investidores sobre a capacidade de receberem todas as obrigações relacionadas aos títulos. No Quadro 2 são apresentadas as classificações das principais agências de risco que atuam no Brasil.

Quadro 2-Classificações das agências de risco

\begin{tabular}{|c|c|c|c|}
\hline Fitch Ratings & Moody's & Standard \& Poor's & Significado na escala \\
\hline AAA & Aaa & AAA & \multirow{7}{*}{$\begin{array}{l}\text { AAA até A- ou A3 } \\
\text { Grau de investimento com } \\
\text { qualidade alta e baixo risco }\end{array}$} \\
\hline $\mathrm{AA}+$ & Aa1 & $\mathrm{AA}+$ & \\
\hline $\mathrm{AA}$ & $\mathrm{Aa} 2$ & $\mathrm{AA}$ & \\
\hline AA- & Aa3 & AA- & \\
\hline $\mathrm{A}+$ & A1 & $\mathrm{A}+$ & \\
\hline A & A2 & A & \\
\hline A- & A3 & A- & \\
\hline $\mathrm{BBB}+$ & Baa1 & $\mathrm{BBB}+$ & \multirow{3}{*}{$\begin{array}{l}\text { BBB+ ou Baa1 até BBB- ou Baa? } \\
\text { Grau de investimento, qualidade } \\
\text { média }\end{array}$} \\
\hline $\mathrm{BBB}$ & Baa2 & $\mathrm{BBB}$ & \\
\hline BBB- & Baa3 & BBB- & \\
\hline $\mathrm{BB}+$ & Ba1 & $\mathrm{BB}+$ & \multirow{6}{*}{$\begin{array}{l}\text { BB+ ou Ba1 até B- ou B3 } \\
\text { Categoria de especulação, baixa } \\
\text { classificação }\end{array}$} \\
\hline $\mathrm{BB}$ & $\mathrm{Ba} 2$ & $\mathrm{BB}$ & \\
\hline BB- & $\mathrm{Ba} 3$ & BB- & \\
\hline $\mathrm{B}+$ & B1 & $\mathrm{B}+$ & \\
\hline $\mathrm{B}$ & B2 & $\mathrm{B}$ & \\
\hline B- & B3 & B- & \\
\hline $\mathrm{CCC}$ & Caa1 & $\mathrm{CCC}+$ & CCC ou Caal até C ou D \\
\hline $\mathrm{CC}$ & Caa2 & $\mathrm{CCC}$ & \multirow{2}{*}{$\begin{array}{l}\text { Risco alto de inadimplência e } \\
\text { baixo interesse }\end{array}$} \\
\hline $\mathrm{C}$ & Caa3 & $\mathrm{CCC}-$ & \\
\hline
\end{tabular}

Revista de Gestão, Finanças e Contabilidade - v. 10, n. 2, p. 22-38, mai./ago. 2020 
Rating para Avaliação de Cooperativas de Crédito: Uma Aplicação do Modelo PEARLS

\begin{tabular}{lll}
$\mathrm{RD}$ & $\mathrm{Ca}$ & $\mathrm{CC}$ \\
$\mathrm{D}$ & $\mathrm{C}$ & $\mathrm{C}$ \\
& & $\mathrm{D}$ \\
\hline
\end{tabular}

Fonte: Elaboração própria.

As classificações triplo A (AAA ou Aaa) até BBB- (Fitch Ratings e Standard \& Poor's) ou A3 (Moody's) para entes federativos e grandes corporações configuram grau de investimento, sinalizando que o risco de crédito é baixo. As classificações BBB+ (Fitch Ratings e Standard \& Poor's) ou Baa1(Moody's) até B- ou B3 (Fitch Ratings e Standard \& Poor's) configuram risco de crédito médio a médio/alto. As classificações abaixo de CCC configuram alto risco de crédito (inadimplência). Alguns fundos de investimento têm em seus estatutos a proibição de investimento em instituições com nível abaixo de B por exemplo.

Do ponto de vista de uma instituição financeira a graduação de risco de um cliente por meio de escores ou rating cumpre duplo papel (LIMA; FONSECA; SILVEIRA; ASSAF NETO, 2018): (i) Como referencial para identificar a chance de perda de uma determinada operação de crédito e, com isso, orientar na precificação do empréstimo ou financiamento; (ii) Atender as exigências das autoridades monetárias do país, que segue a tendência mundial de utilização de sistemas de classificação de risco (escores e ratings) como forma de acompanhar a qualidade da carteira da instituição financeira e, consequentemente, orientar $o$ provisionamento dos créditos de liquidação duvidosa.

\subsection{MODELO PEARLS}

Os principais modelos de análise econômico-financeira e rating utilizados pelas cooperativas de crédito foram analisados por Vasconcelos (2006). O autor analisou os modelos: alerta Temprana; PEARLS; sinalizadores e indicadores criados por analistas do Banco Central do Brasil; sistema de classificação de risco do Unicred; sistema de análise do Sicredi (SAS); programa de análise financeira e de risco do Sicoob (PROAF) e; monitoramento on-Line da Cecremge.

Segundo o autor o PEARLS é reconhecido mundialmente como um dos modelos mais abrangentes e eficazes para a análise econômico-financeira das cooperativas. Esse modelo, com devidas adaptações para adequar-se à estrutura contábil do Brasil, pode ser utilizado para a análise de cooperativas de crédito brasileiras.

O modelo PEARLS foi desenvolvido pelo Conselho Mundial de Cooperativismo de Poupança e Crédito (WOCCU - World Council of Credit Unions) no final dos anos 1980 como uma adaptação do método CAMELS para as cooperativas de crédito (BRESSAN; BRAGA; BRESSAN; RESENDE FILHO, 2010) - a metodologia CAMELS foi criada pelo Banco Central Americano (Federal Reserve) para avaliar as instituições financeiras dos EUA, servindo como base para a criação de ratings (CARREIRO; CUNHA, 2008).

Para Richardson (2009), o PEARLS é um instrumento de gestão para as cooperativas que também pode ser usado como uma ferramenta de supervisão pelos reguladores. Como ferramenta de gestão, o PEARLS apresenta sinais para os gestores antes que os problemas se tornem prejudiciais. Para os conselhos de administração, o PEARLS fornece uma ferramenta para monitorar o progresso da administração em direção a objetivos financeiros. Para os reguladores, o PEARLS oferece indicadores e padrões para supervisionar o desempenho econômico-financeiro das cooperativas de crédito.

O PEARLS é um modelo composto por um conjunto de 39 indicadores financeiros baseado em seis pilares:

$\mathbf{P}$ - protection (proteção): avaliação da proteção adequada implantada na cooperativa em comparação com a provisão para perdas de crédito;

E - effective financial structure (estrutura financeira efetiva): avaliação de ativos, 
exigibilidades e capital. O WOCCU recomenda manter de $70 \%$ a $80 \%$ do total de ativos em carteiras de crédito, pois apresentam margens superiores aos rendimentos obtidos em outros investimentos;

A - assets quality (qualidade dos ativos): indicadores de impacto dos ativos não lucrativos. A WOCCU recomenda financiar a totalidade dos ativos não-lucrativos (não financeiros) com o capital social da cooperativa ou com outros passivos com baixo custo financeiro;

$\mathbf{R}$ - rates of return and costs (taxas de retorno e custos): indicadores que permitem avaliar a composição das rendas líquidas. Auxiliam no acompanhamento dos rendimentos dos investimentos e no gerenciamentos das despesas operacionais. Os indicadores permitem avaliar qual a eficiência da cooperativa na alocação de recursos em investimentos que produzem maiores margens;

$\mathbf{L}$ - Liquidity (liquidez): quando a estrutura financeira da cooperativa, que tem por base as quotas dos associados, é trocada por depósitos voláteis, a gestão eficiente da liquidez é essencial. De forma tradicional, a liquidez é entendida como o caixa disponível para empréstimo - um fator cujo controle é exclusivo da cooperativa. A partir da instauração de sacáveis como depósitos de poupança, a ideia de liquidez é alterada, referindo-se ao caixa necessário para retirada - variável que não é controlada pela cooperativa. Neste caso as reservas de liquidez são fundamentais, embora elas impliquem também em perda de custo de oportunidade;

$\mathbf{S}$ - signs of growth (sinais de crescimento): permite avaliar a valorização sustentável dos ativos devido ao seu crescimento e rentabilidade. O PEARLS vincula o crescimento à rentabilidade e a outras áreas-chave, avaliando a força do sistema como um todo.

O detalhamento dos indicadores, com suas fórmulas e objetivos, pode ser encontrado nos trabalhos de Richardson (2009) e Bressan, Braga, Bressan e Resende Filho (2011).

\subsection{METODOLOGIA DE AVALIAÇÃO DE RISCO DO FGCOOP}

O Fundo Garantidor do Cooperativismo de Crédito (FGCOOP) tem por objetivo prestar garantia de créditos contra as instituições associadas nas situações de decretação da intervenção ou da liquidação extrajudicial (FGCOOP, 2017). O fundo tem por principal finalidade proteger depositantes e investidores das instituições associadas até o valor de $\mathrm{R} \$ 250 \mathrm{mil}$ por $\mathrm{CPF} / \mathrm{CNPJ}$, com destaque para os depósitos à vista e a prazo, depósitos de poupança, letras de crédito do agronegócio e letras de crédito imobiliário. Também visa contribuir para prevenção de crise sistêmica no segmento cooperativista e contribuir para a manutenção da estabilidade do Sistema Nacional de Crédito Cooperativo (SNCC).

O FGCOOP adota um modelo de monitoramento e avaliação dos riscos financeiros das cooperativas com o objetivo de avaliar o grau de risco de descontinuidade ("quebra") das cooperativas associadas ao fundo, antecipar problemas, auxiliar na busca de solução sistêmica e preservar o patrimônio do fundo evitando a necessidade de cobertura dos depósitos. O modelo calcula um rating que é segregado em duas análises, uma quantitativa $(80 \%)$ e outra qualitativa $(20 \%)$

A Tabela 1 apresenta os indicadores, as contas COSIF (Plano Contábil das Instituições do Sistema Financeiro Nacional), a memória de cálculo e os pesos de cada indicador utilizado na análise quantitativa. O resultado da análise deriva da classificação do nível de risco (após o cálculo do indicador) multiplicado pelo peso do indicador.

$\mathrm{Na}$ análise qualitativa são avaliadas a forma de associação e a tradição e imagem da cooperativa singular. Referente à forma de associação (que representa $70 \%$ dos $20 \%$ ) é verificado se a cooperativa de crédito singular é associada a uma Confederação, Central ou se a mesma não é associada a uma Cooperativa Central. Em relação à tradição e imagem da singular (30\% restantes dos 20\%) é verificada a evolução dos depósitos nos últimos doze meses, 
Huscher, Moreira e Silva (2020)

Rating para Avaliação de Cooperativas de Crédito: Uma Aplicação do Modelo PEARLS

sendo uma forma de verificar a credibilidade da cooperativa perante seus associados e a comunidade onde atua.

Após a mensuração da análise quantitativa e qualitativa é apurada a pontuação final, resultante da ponderação de $80 \%$ da análise quantitativa e $20 \%$ da qualitativa. Com o resultado da pontuação final é apurado o risco conforme apresentado na Tabela 2.

Tabela 1 - Matriz de análise quantitativa do FGCOOP

\begin{tabular}{c|c|c|c|c|c|c}
\hline \multirow{2}{*}{ Peso } & Indicadores & \multicolumn{5}{c}{ Níveis de Risco } \\
\cline { 3 - 6 } & Índice de Endividamento & 1 & 2 & 3 & 4 & 5 \\
\hline 15 & $4000000 / P R$ & $<=4$ & $>4 \mathrm{e}$ & $>4 \mathrm{e}$ & $>8 \mathrm{e}$ & $>10$ \\
& & & $<=6$ & $<=8$ & $<=10$ \\
& & & & & \\
\hline 15 & Índice de Alavancagem & $<=4$ & $>4 \mathrm{e}$ & $>4 \mathrm{e}$ & $>8 \mathrm{e}$ & $>10$ \\
& $(160000001-16900008) / \mathrm{PR}$ & & $<=6$ & $<=8$ & $<=10$ &
\end{tabular}

\begin{tabular}{|c|c|c|c|c|c|c|}
\hline 15 & $\begin{array}{c}\text { Índice de Liquidez } \\
(11000006+12000005+13000004 \\
+14500008- \\
\text { Vinculos }) /(41000007+44500009) * 100\end{array}$ & $>=40 \%$ & $\begin{array}{c}>=30 \% \\
\quad \mathrm{e} \\
<40 \%\end{array}$ & $\begin{aligned}> & =20 \% \\
& \mathrm{e} \\
< & 30 \%\end{aligned}$ & $\begin{array}{l}>=10 \% \\
\quad \mathrm{e} \\
<20 \%\end{array}$ & $10 \%$ \\
\hline 10 & $\begin{array}{c}\text { Rentabilidade sobre PR } \\
\left\{\left[\left(70000009-800000061^{\circ}\right.\right.\right. \\
\text { sem })+\left(70000009-80000002^{\circ}\right. \\
\text { sem })] / \mathrm{mr}\}^{*} 12 /(\mathrm{PR}-\text { último mês exercício }\end{array}$ & $>=9 \%$ & $\begin{array}{l}>=6 \% \\
\quad \mathrm{e} \\
<9 \%\end{array}$ & $\begin{array}{c}>=3 \% \\
\mathrm{e} \\
<6 \%\end{array}$ & $\begin{array}{c}>=1 \% \\
\mathrm{e} \\
<3 \%\end{array}$ & $<1 \%$ \\
\hline
\end{tabular}
anterior)*100

\begin{tabular}{|c|c|c|c|c|c|c|c|}
\hline \multirow[t]{2}{*}{10} & \multirow{2}{*}{$\begin{array}{l}\text { Concentração } \\
\text { de Ativos }\end{array}$} & \multirow{2}{*}{$\begin{array}{c}10>\text { tomadores/ } \\
(16000001- \\
16900008)]^{*} 100 \\
\text { (Maior Tomador/ } \\
\text { PR)*100 }\end{array}$} & \multirow{2}{*}{$\begin{array}{l}<=20 \% \\
<=5,0 \%\end{array}$} & \multirow{2}{*}{$\begin{array}{l}>20 \% \mathrm{e} \\
<=35 \% \\
\\
>5 \% \mathrm{e} \\
<=10 \%\end{array}$} & \multirow{2}{*}{$\begin{array}{l}>35 \% \mathrm{e} \\
<=50 \% \\
\\
>10 \% \mathrm{e} \\
<=20 \%\end{array}$} & \multirow{2}{*}{$\begin{array}{l}>50 \% \mathrm{e} \\
<=65 \% \\
\\
>20 \% \mathrm{e} \\
<=30 \%\end{array}$} & \multirow{2}{*}{$\begin{array}{l}>65 \% \\
<30 \%\end{array}$} \\
\hline & & & & & & & \\
\hline 10 & \multicolumn{2}{|c|}{$\begin{array}{c}\text { Concentração de Passivos } \\
{[>\text { depositantes/ }} \\
(41000007+44500009)] * 100\end{array}$} & $<=20 \%$ & $\begin{array}{l}>20 \% \mathrm{e} \\
<=35 \%\end{array}$ & $\begin{array}{l}>35 \% \mathrm{e} \\
<=50 \%\end{array}$ & $\begin{array}{l}>50 \% \mathrm{e} \\
<=65 \%\end{array}$ & $>65 \%$ \\
\hline 20 & \multicolumn{2}{|c|}{ Índice de Basiléia } & $>=40 \%$ & $\begin{array}{l}>=30 \% \\
\mathrm{e}<40 \%\end{array}$ & $\begin{array}{l}>=20 \% \\
\mathrm{e}<30 \%\end{array}$ & $\begin{array}{l}>=15 \% \\
\mathrm{e}<20 \%\end{array}$ & $<15 \%$ \\
\hline 5 & \multicolumn{2}{|c|}{$\begin{array}{c}\text { Índice de Provisionamento } \\
{[169000008 /(16000001-16900008)]^{*}-100}\end{array}$} & $<=3 \%$ & $\begin{array}{l}>3 \% \mathrm{e} \\
<=6 \%\end{array}$ & $\begin{array}{l}>6 \% \mathrm{e} \\
<=9 \%\end{array}$ & $\begin{array}{l}>9 \% \mathrm{e} \\
<=12 \%\end{array}$ & $>12 \%$ \\
\hline
\end{tabular}

Fonte: FGCOOP (2017)

Tabela 2 - Apuração do risco FGCOOP

\begin{tabular}{c|c|c|c|c|c|c|c|c|c}
\hline Total de Pontos & $\begin{array}{c}100 \mathrm{a} \\
144\end{array}$ & $\begin{array}{c}145 \mathrm{a} \\
189\end{array}$ & $\begin{array}{c}190 \mathrm{a} \\
234\end{array}$ & $\begin{array}{c}235 \mathrm{a} \\
279\end{array}$ & $\begin{array}{c}280 \mathrm{a} \\
324\end{array}$ & $\begin{array}{c}325 \mathrm{a} \\
368\end{array}$ & $\begin{array}{c}369 \mathrm{a} \\
412\end{array}$ & $\begin{array}{c}413 \mathrm{a} \\
456\end{array}$ & $\begin{array}{c}457 \mathrm{a} \\
500\end{array}$ \\
\hline $\begin{array}{c}\text { Classificação de Risco - Res. } \\
2.682\end{array}$ & AA & A & B & C & D & E & F & G & H \\
\hline
\end{tabular}

Fonte: FGCOOP (2017) 
Huscher, Moreira e Silva (2020)

Rating para Avaliação de Cooperativas de Crédito: Uma Aplicação do Modelo PEARLS

\section{PROCEDIMENTOS METODOLÓGICOS}

Este estudo se baseou em dados secundários disponibilizados pelo Banco Central do Brasil a partir dos relatórios do sistema IF Data - Dados Selecionados de Entidades Supervisionadas e os Balancetes 4010 no período de junho de 2014, dezembro de 2014, junho de 2015 e dezembro de 2015.

Do total de 1.060 cooperativas de crédito singulares no Brasil no ano de 2015, foram selecionadas 212 cooperativas que tiveram análises de crédito efetuadas pelo Banco X no ano de 2015. Dessas, 193 contraíram crédito por meio de cartão de crédito (modalidade na qual a disponibilização do cartão de crédito aos Cooperados é realizada por parte do Banco emissor do cartão de crédito) e 147 por meio de repasse para crédito rural. Esse conjunto de dados contemplou cooperativas de crédito singulares independentes e de todos os sistemas cooperativos (SICOOB, SICREDI, CECRED, UNICRED, CRESOL, UNIPRIME, CECRERS, CREDISIS) localizadas em diversas regiões do Brasil. Embora as cooperativas tenham características diversas, como cooperados produtores rurais ou cooperados que se assemelham a clientes normais de bancos múltiplos, o cálculo dos indicadores uniformiza a análise do desempenho e risco financeiro, possibilitando assim a comparação.

Os indicadores usados para avaliar o desempenho econômico-financeiro e considerados como variáveis explicativas para o modelo, foram calculados de acordo com o modelo PEARLS. Para tanto, foi necessário adequar os indicadores ao Plano Contábil das Instituições Financeiras do Sistema Financeiro Nacional - COSIF. Considerando que as Cooperativas têm seus dados disponíveis no sistema de informações do Banco Central e que esse sistema traz as contas do COSIF “abertas” até o nível 3, foi possível calcular 23 dos 39 indicadores do modelo PEARLS. No Quadro 3 são listados os indicadores calculados e sua descrição.

Quadro 3 - Indicadores financeiros calculados

\begin{tabular}{|c|c|c|c|}
\hline \multicolumn{2}{|r|}{ Pilar } & Indicador & Descrição \\
\hline $\mathrm{P}$ & Proteção & $\begin{array}{l}\text { P1 = Provisão para liquidação duvidosa sob } \\
\text { operações de crédito / Carteira Total. } \\
\text { P3 = Operações de Risco nível D até H / } \\
\text { Classificação da carteira de créditos. } \\
\text { P4 = Operações de Risco nível D até H - } \\
\text { Percentual de Provisão Estimado nível D } \\
\text { até H / Patrimônio Líquido Ajustado. }\end{array}$ & $\begin{array}{l}\text { Volume de provisão de créditos de liquidação } \\
\text { duvidosa em relação à carteira total. } \\
\text { Parcela da carteira de crédito classificada com } \\
\text { nível de risco superior a } 61 \text { dias de atraso. } \\
\text { Parcela da carteira de crédito classificada com } \\
\text { nível de risco superior a } 61 \text { dias de atraso não } \\
\text { provisionada em relação ao PLA. }\end{array}$ \\
\hline $\mathrm{E}$ & $\begin{array}{c}\text { Estrutura } \\
\text { financeira } \\
\text { efetiva }\end{array}$ & $\begin{array}{l}\text { E1 = Operações de crédito líquidas / Ativo } \\
\text { Total. } \\
\text { E2 = Investimentos / Ativo Total. } \\
\text { E3 = Capital Social / Ativo Total. } \\
\text { E6 = Ativo Total / Patrimônio Líquido } \\
\text { Ajustado. }\end{array}$ & $\begin{array}{l}\% \text { do ativo total investido na carteira de crédito } \\
\text { da cooperativa. } \\
\% \text { do ativo total investido em ativos financeiros. } \\
\% \text { do ativo financiado pelos cooperados. } \\
\text { Recursos próprios no financiamento dos ativos. } \\
\text { Indicador de alavancagem. }\end{array}$ \\
\hline A & $\begin{array}{l}\text { Qualidade } \\
\text { dos ativos }\end{array}$ & $\begin{array}{l}\text { A2 = Imobilização = Ativo Permanente / } \\
\text { Patrimônio Líquido Ajustado. } \\
\text { A4 = Depósitos totais / Ativo total. }\end{array}$ & $\begin{array}{l}\text { Pela Resolução } 2.669 / 99 \text { do BACEN, o total dos } \\
\text { recursos aplicados no Ativo Permanente não } \\
\text { pode ultrapassar } 50 \% \text { do valor do patrimônio } \\
\text { líquido. } \\
\text { Total dos ativos que provêm de depósitos. }\end{array}$ \\
\hline $\mathrm{R}$ & $\begin{array}{l}\text { Taxas de } \\
\text { retorno e } \\
\text { custos }\end{array}$ & $\begin{array}{l}\text { R1 = Rendas de crédito / Operações de } \\
\text { crédito média. } \\
\text { R4 = Despesas de Obrigações por } \\
\text { empréstimos e repasses / Obrigações por } \\
\text { empréstimos e repasses médio. }\end{array}$ & Custo dos fundos de empréstimos. \\
\hline
\end{tabular}


Huscher, Moreira e Silva (2020)

Rating para Avaliação de Cooperativas de Crédito: Uma Aplicação do Modelo PEARLS

\begin{tabular}{|c|c|c|c|}
\hline & & $\begin{array}{l}\text { R5 = Margem Bruta / Ativo Total Médio. } \\
\text { R6 = Despesas Operacionais / Ativo Total } \\
\text { Médio. } \\
\text { R11 = Rendas de prestação de serviços / } \\
\text { Despesas administrativas. } \\
\text { R13 = Despesas Administrativas / Ativo } \\
\text { Total Médio. }\end{array}$ & $\begin{array}{l}\text { Margem de renda bruta gerada em relação ao } \\
\text { ativo total médio. } \\
\text { Custo associado com o gerenciamento de todos } \\
\text { os ativos da cooperativa de crédito, indicando o } \\
\text { grau de eficiência ou ineficiência operacional. } \\
\% \text { das despesas administrativas cobertas pelas } \\
\text { receitas de prestação de serviços. } \\
\% \text { das despesas administrativas em relação ao } \\
\text { ativo total. }\end{array}$ \\
\hline $\mathrm{L}$ & Liquidez & $\begin{array}{l}\text { L1 = Disponibilidades / Depósitos à Vista. } \\
\text { L2 = Ativos curto prazo/ Depósitos totais. }\end{array}$ & $\begin{array}{l}\text { Capacidade da cooperativa em satisfazer seus } \\
\text { compromissos imediatos, pois ambas as contas } \\
\text { são de curto prazo. } \\
\text { Proxy para a liquidez corrente. }\end{array}$ \\
\hline$S$ & $\begin{array}{c}\text { Sinais de } \\
\text { crescimento }\end{array}$ & $\begin{array}{l}\text { S1 = (Rec. Operac. mês corrente / Rec. } \\
\text { Operac. do mês anterior })-1 . \\
\text { S3 = (Oper. D-H do mês corrente / Oper. D- } \\
\text { H do mês anterior })-1 . \\
\text { S6 = (desp. adm. mês corrente / desp. adm. } \\
\text { do mês anterior) }-1 . \\
\text { S7 = (PLA do mês corrente / PLA do mês } \\
\text { anterior) - } 1 . \\
\text { S8 = (AT do mês corrente / AT do mês } \\
\text { anterior) - } 1 . \\
\text { S9 = (Oper. crédito do mês corrente / Oper. } \\
\text { crédito do mês anterior })-1 .\end{array}$ & $\begin{array}{l}\text { Taxa de crescimento da receita operacional. } \\
\text { Taxa de crescimento das operações de crédito } \\
\text { com nível de risco D-H. } \\
\text { Taxa de crescimento das despesas } \\
\text { administrativas. } \\
\text { Taxa de crescimento do Patrimônio Líquido } \\
\text { Ajustado (PLA). } \\
\text { Taxa de crescimento do Ativo Total (AT). } \\
\text { Crescimento das operações de crédito. Quanto } \\
\text { maior o índice, mais a instituição está } \\
\text { expandindo as operações de crédito. }\end{array}$ \\
\hline
\end{tabular}

Fonte: Bressan et al. (2011).

Os indicadores financeiros do grupo S - Signs of Growth (Sinais de Crescimento) foram calculados anualmente, baseado no exercício de 2015 em relação a 2014. Adicionalmente foi incluído o índice de Basileia (IB) definido pelo Comitê de Basileia e adotado pelo Banco Central do Brasil. Esse indicador é disponibilizado no Relatório IF Data publicado pelo Bacen.

Para garantir a ausência de multicolinearidade entre as variáveis explicativas (indicadores financeiros do modelo PEARLS), foi efetuada uma análise fatorial exploratória por meio da análise de componentes principais. Os indicadores selecionados, além do Índice Basileia, são apresentados no Quadro 4.

Quadro 4 - Indicadores financeiros selecionados após a análise fatorial

\begin{tabular}{cl}
\hline Pilar do PEARLS & Indicadores \\
\hline P & P1 e P3 \\
E & E1 e E2 \\
A & A2 \\
R & R13 \\
L & L1 e L2 \\
S & S1 \\
\hline
\end{tabular}

Fonte: Dados da pesquisa.

Esta pesquisa aborda um problema de classificação, uma vez que se busca categorizar as cooperativas em função do risco de crédito que representam a outros agentes. A técnica utilizada para o problema de classificação, visando o desenvolvimento do modelo de rating, foi o modelo logit multinomial, também denominado de regressão logística multinomial, usando o método de máxima verossimilhança. De acordo com Fávero e Belfiori (2017) a regressão logística é uma das técnicas mais utilizadas no tratamento de problemas de classificação. Neste estudo o problema de classificação é policotômico, isto é, busca-se classificar as observações 
dentre as finitas classes previamente estabelecidas (mais que duas). Assim, o fenômeno em estudo oferece mais de duas possibilidades de resposta, o que, segundo Fávero e Belfiori (2017), determina a necessidade de uso de uma regressão logística multinomial.

Em um primeiro momento é necessário definir as categorias iniciais de análise, que são as variáveis resposta do modelo indicando as classes prévias de rating. Para esta tarefa a pesquisa utilizou a classificação usada pelo FGCOOP. Tal estratégia foi utilizada pela não observância, no banco de dados inicial, de uma classificação prévia no conjunto de dados de supervisão.

Cabe ressaltar que no modelo de regressão logística multinomial estimado, a classe à qual a ocorrência observada pertencerá é definida com base na maior probabilidade de pertencimento de acordo com os resultados obtidos pelas funções logit estimadas. A função logit é o logaritmo natural da razão de chance. O modelo logístico multinomial estima coeficientes para $\mathrm{k}-1$ funções logit ( $\mathrm{k}=$ número de classes da variável resposta).

Esse procedimento difere do modelo de regressão logística binária, na qual se estabelece um ponto de corte para as classes. Uma prática que é questionável tecnicamente, para as instituições financeiras que usam regressão logística, é estimar múltiplos pontos de corte ao longo das probabilidades calculadas para um modelo de regressão logística binária ao invés de utilizar o modelo de regressão logística multinomial.

Após a classificação das observações de acordo com o modelo multinomial, a eficiência global do modelo é, em geral, estimada pela medida de acurácia total - ACC, definida pela expressão 1:

$$
A C C=\frac{T P+T N}{T P+T N+F P+F N}
$$

Em que TP significa "verdadeiro positivo", TN "verdadeiro negativo", FP "falso positivo" e FN "falso negativo". Em uma matriz de classificação (matriz de confusão) essa medida se dá pela soma da diagonal principal dividida pela soma da matriz.

Para a estimação e posterior validação cruzada (cross validation) do modelo, a amostra foi particionada aleatoriamente em 2 partes. A subamostra de estimação (treinamento) foi formada por 141 cooperativas ( $2 / 3$ da amostra total). A subamostra de validação (teste) foi formada por 71 cooperativas ( $1 / 3$ da amostra). Este procedimento está em conformidade com o que é sugerido por Hair Jr. et al. (2009) para estimação e validação de modelos de regressão.

Com relação a possível influência de variáveis endógenas no modelo, é relevante considerar que os ratings são estimados após a observação do desempenho financeiro das cooperativas, ou seja, são estimados ao final do exercício. Nesse caso, considera-se que o desempenho seja afetado pelas condições macro e microeconômicas do período de análise, e não pelo próprio rating. Logo, não há simultaneidade na relação entre o rating e as variáveis explicativas do modelo. No entanto, conforme ressaltam Lima, Fonseca, Silveira e Assaf Neto (2018), abordagens alternativas para problemas associados à presença de variáveis endógenas consistem em tema relevante de pesquisa. No caso de regressões logísticas, os métodos de identificação de variáveis instrumentais (VI) e a multiple indicator solution (MIS) são abordagens alternativas para lidar com o problema de endogeneidade causado pela presença de variáveis omitidas (GUEVARA; POLANCO, 2016).

\section{RESULTADOS E DISCUSSÃO}

A classificação prévia das 212 cooperativas da amostra por nível de risco (classe de rating) foi feita segundo a metodologia do FGCOOP. A Tabela 3 apresenta a distribuição das cooperativas para cada nível, representando o rating prévio gerado pelo Fundo Garantidor. 
Huscher, Moreira e Silva (2020)

Rating para Avaliação de Cooperativas de Crédito: Uma Aplicação do Modelo PEARLS

Tabela 3 - Classificação prévia por nível de risco

\begin{tabular}{c|r|r}
\hline Nível de risco & Quantidade & $\%$ \\
\hline A & 39 & $18,4 \%$ \\
B & 96 & $45,3 \%$ \\
C & 51 & $24,1 \%$ \\
D & 15 & $7,1 \%$ \\
E & 8 & $3,8 \%$ \\
F & 3 & $1,4 \%$ \\
Total & 212 & $100 \%$ \\
\hline
\end{tabular}

Fonte: Dados da pesquisa.

Verifica-se seis níveis de risco para as cooperativas de crédito consideradas na amostra. Considerando que a metodologia de classificação do FGCOOP utiliza nove níveis possíveis (AA, A, B , C , D, E, F, G, H) e na amostra apurou-se apenas seis níveis, é possível verificar que dentre as cooperativas amostradas nenhuma foi classificada com o maior nível (AA) e nem com os dois últimos piores níveis $(\mathrm{G}$ e $\mathrm{H})$. As classes com maior e menor frequência foram, respectivamente, as classes B e F. É possível também notar concentração nas classes A, B e C que representam $87,74 \%$ das observações.

Pelo critério de parcimônia em conjunto com o ajuste geral alcançado no modelo de regressão multinomial, considerou-se mais adequado a segregação da base em quatro níveis de riscos. O princípio da parcimônia consiste em produzir modelos mais simples em detrimento aos mais complexos (BALIOS; THOMADAKIS; TSIPOURI, 2016). Por conta disso, as classes maiores (A e B) foram agregadas na classe A e as menores (E e F) na classe D. A Tabela 4 apresenta as frequências da reclassificação prévia das cooperativas nos 4 níveis de risco, compondo assim os níveis da variável resposta do modelo.

Tabela 4 - Reclassificação prévia por nível de risco

\begin{tabular}{crr}
\hline Nível de risco & Quantidade & $\%$ \\
\hline A & 135 & $63,7 \%$ \\
B & 51 & $24,1 \%$ \\
C & 15 & $7,1 \%$ \\
D & 11 & $5,2 \%$ \\
Total & 212 & $100 \%$ \\
\hline
\end{tabular}

Fonte: Dados da pesquisa.

O modelo foi estimado na amostra de treinamento e a seleção das variáveis foi feita por meio da técnica stepwise para garantir somente a inclusão de variáveis estatisticamente significativas. O modelo final foi obtido com pelo menos um indicador financeiro (variável explicativa) de cada pilar do PEARLS, com exceção do pilar S, presente em todas as equações do modelo. O índice de Basileia não apresentou significância e foi desconsiderado no modelo final. A Tabela 5 apresenta os resultados dos testes de razão de verossimilhança para o ajuste geral do modelo e significância estatística dos coeficientes. Na tabela também é possível verificar as variáveis explicativas que compuseram o modelo final (P1, E1, A2, R5 e L2).

Tabela 5 - Ajuste do modelo

\begin{tabular}{lrrr}
\hline & Qui-quadrado & df & Sig. \\
\hline Razão de verossimilhança geral do modelo & 168,75 & 15 & 0,000 \\
Razão de verossimilhança dos coeficientes & & & \\
Intercepto & 16,316 & 3 &, 001 \\
P1 & 11,964 & 3 &, 008 \\
E1 & 15,795 & 3 &, 001 \\
A2 & 28,689 & 3 &, 000 \\
R5 & 44,699 & 3 &, 000 \\
L2 & 9,050 & 3 &, 029 \\
\hline
\end{tabular}

Fonte: Dados da pesquisa. 
A razão de verossimilhança geral, com valor de significância igual a 0,000 , indica que no modelo estimado pelo menos uma variável explicativa (indicador financeiro) é significante para explicar a probabilidade de ocorrência de pelo menos um dos eventos (classes de risco do rating). Individualmente, para cada coeficiente estimado no modelo, também é possível verificar significância a pelo menos $5 \%$. Ou seja, os coeficientes apresentam significância em pelo menos uma das funções geradas pelo modelo logístico multinomial.

O poder de explicação geral do modelo pode ser avaliado pelos valores pseudo Rquadrado, que se assemelham ao coeficiente de determinação em modelos de regressão linear (quanto maior melhor). O valor resultante do Cox e Snell foi de 0,698, o de McFadden foi de 0,539 e o de Nagelkerke foi de 0,783 . Destes valores pseudo R-quadrado, o mais intuitivo para interpretação é o Nagelkerke, que varia de 0 a 1 . Neste caso, o poder de explicação da variabilidade da razão de probabilidade foi de $78,3 \%$, indicando um bom ajuste do modelo aos dados da amostra.

A Tabela 6 apresenta os coeficientes beta (B) estimados para as funções logit do modelo e o teste Wald de significância. É possível verificar que todas as variáveis têm coeficientes significativos a $5 \%$ em pelo menos uma das funções do modelo logístico.

\begin{tabular}{|c|c|c|c|c|c|c|c|c|c|}
\hline \multirow{2}{*}{\multicolumn{2}{|c|}{$\begin{array}{l}\text { Riscos } \\
(\mathrm{D}=\text { categoria } \\
\text { de referência) }\end{array}$}} & \multirow[t]{2}{*}{ B } & \multirow{2}{*}{$\begin{array}{r}\text { Erro } \\
\text { padrão }\end{array}$} & \multirow[t]{2}{*}{ Wald } & \multirow[t]{2}{*}{$\mathrm{df}$} & \multirow[t]{2}{*}{ Sig. } & \multirow[t]{2}{*}{$\operatorname{Exp}(B)$} & \multicolumn{2}{|c|}{$\begin{array}{c}\text { 95\% Intervalo de confiança } \\
\text { para Exp (B) }\end{array}$} \\
\hline & & & & & & & & Limite Inf & Limite Sup \\
\hline \multirow{6}{*}{ A } & Intercept & 35,862 & 15,090 & 5,648 & 1 & 017 & & & \\
\hline & $\mathrm{P} 1$ & $-74,541$ & 28,053 & 7,061 & 1 & ,008 & $4,238 \mathrm{E}-033$ & $5,606 \mathrm{E}-057$ & 3,204E-009 \\
\hline & E1 & $-32,994$ & 14,290 & 5,331 & 1 & 021 & $4,686 \mathrm{E}-015$ & $3,214 \mathrm{E}-027$ & ,007 \\
\hline & $\mathrm{A} 2$ & $-28,141$ & 9,585 & 8,619 & 1 & ,003 & $6,003 \mathrm{E} 013$ & $4,161 \mathrm{E}-021$ & $8,661 \mathrm{E}-021$ \\
\hline & R5 & $\begin{array}{r}225,13 \\
7\end{array}$ & 55,909 & 16,215 & 1 & ,000 & $5,969 \mathrm{E}+097$ & $\begin{array}{r}1,534 \mathrm{E}+05 \\
0\end{array}$ & $2,322 \mathrm{E}+145$ \\
\hline & $\mathrm{L} 2$ & 9,394 & 4,911 & 3,659 & 1 & ,056 & 12016,892 & ,794 & 181939915,6 \\
\hline \multirow{6}{*}{ B } & Intercept & 36,612 & 15,011 & 5,949 & 1 & ,015 & & & \\
\hline & $\mathrm{P} 1$ & $-70,872$ & 27,739 & 6,528 & 1 & 011 & 1663E-031 & $4,069 \mathrm{E}-055$ & $6,797 \mathrm{E}-008$ \\
\hline & E1 & $-27,403$ & 14,198 & 3,725 & 1 & ,054 & $1,256 \mathrm{E}-012$ & $1,032 \mathrm{E}-024$ & 1,529 \\
\hline & A2 & $-33,807$ & 9,771 & 11,971 & 1 & ,001 & $2,079 \mathrm{E}-015$ & $1,002 \mathrm{E}-023$ & $4,314 \mathrm{E}-007$ \\
\hline & R5 & 46,085 & 44,298 & 1,082 & 1 & ,298 & $1,034 \mathrm{E}+20$ & $2,032 \mathrm{E}-018$ & $5,265 \mathrm{E}+057$ \\
\hline & L2 & 7,244 & 4,874 & 2,209 & 1 & 137 & 1400,123 & ,099 & 19737796,25 \\
\hline \multirow{6}{*}{$\mathrm{C}$} & Intercept & 28,111 & 14,347 & 3,839 & 1 & ,050 & & & \\
\hline & P1 & $-53,778$ & 22,651 & 5,637 & 1 & ,018 & $4,409 \mathrm{E}-024$ & $2,311 \mathrm{E}-043$ & $8,412 \mathrm{E}-005$ \\
\hline & E1 & $-29,145$ & 14,040 & 4,309 & 1 & ,038 & $2,200 \mathrm{E}-013$ & $2,463 \mathrm{E}-025$ & ,197 \\
\hline & A2 & $-12,645$ & 7,379 & 2,937 & 1 & ,087 & $3,222 \mathrm{E}-006$ & $1,687 \mathrm{E}-012$ & 6,154 \\
\hline & R5 & $-1,249$ & 16,560 & ,006 & 1 & 940 & ,287 & $2,300 \mathrm{E}-015$ & $3,578 \mathrm{E}+13$ \\
\hline & L2 & 8,145 & 4,712 & 2,988 & 1 &, 084 & 3446,767 &, 336 & 35364802,74 \\
\hline
\end{tabular}

Fonte: Dados da pesquisa.

Considerando os coeficientes estimados, é possível descrever as funções logit do modelo multinomial conforme as expressões 2,3 e 4 .

$$
\begin{aligned}
& f 1=35,862-74.541 \cdot P 1-32,994 \cdot E 1-28,141 \cdot A 2+225,137 \cdot R 5+9,394 \cdot L 2 \\
& f 2=36,612-70,872 \cdot P 1-27,403 \cdot E 1-33,807 \cdot A 2+46,085 \cdot R 5+7,244 \cdot L 2 \\
& f 3=28,111-53,778 \cdot P 1-29,145 \cdot E 1-12,645 \cdot A 2-1,249 \cdot R 5+8,145 \cdot L 2
\end{aligned}
$$

As probabilidades associadas a chance de uma cooperativa ser classificada em cada nível de risco X, sendo A: baixo risco, B: médio baixo risco, C: médio risco e, D: alto risco, podem ser calculadas, respectivamente, pelas expressões 5, 6, 7 e 8 . 
Rating para Avaliação de Cooperativas de Crédito: Uma Aplicação do Modelo PEARLS

$$
\begin{aligned}
& P(X=A)=\frac{e^{f 1}}{1+e^{f 1}+e^{f 2}+e^{f 3}} \\
& P(X=B)=\frac{e^{f 2}}{1+e^{f 1}+e^{f 2}+e^{f 3}} \\
& P(X=C)=\frac{e^{f 3}}{1+e^{f 1}+e^{f 2}+e^{f 3}} \\
& P(X=D)=\frac{1}{1+e^{f 1}+e^{f 2}+e^{f 3}}
\end{aligned}
$$

Apenas como exemplo, considerando uma Cooperativa de Crédito com os seguintes indicadores: $\mathrm{P} 1=0,1124, \mathrm{E} 1=0,7333, \mathrm{~A} 2=0,3034, \mathrm{R} 5=0,0260$ e $\mathrm{L} 2=0,7701$ e aplicando as expressões acima, obtêm-se as seguintes probabilidades: $\mathrm{P}(\mathrm{A})=0,9327, \mathrm{P}(\mathrm{B})=0,0587, \mathrm{P}(\mathrm{C})=$ 0,0082 e $P(D)=0,0004$. Nesse caso a cooperativa com tais indicadores financeiros seria classificada como nível "A" de risco.

Para verificar a validade do modelo considerando o seu poder preditivo, o modelo foi ajustado aos dados de treinamento gerando as informações trazidas na matriz de confusão disponível na Tabela 7.

Tabela 7 - Poder preditivo do modelo

\begin{tabular}{c|rrrrrrr}
\hline \multirow{2}{*}{ Observado } & \multicolumn{7}{|c|}{ Classificação Prevista } \\
\cline { 2 - 7 } & \multicolumn{1}{c|}{ A } & \multicolumn{1}{c|}{ B } & C & D & Percentual de acerto \\
\hline A & 71 & 7 & 1 & 0 & $89,9 \%$ \\
B & 12 & 24 & 0 & 0 & $66,7 \%$ \\
C & 0 & 5 & 8 & 2 & $53,3 \%$ \\
D & 0 & 0 & 1 & 10 & $90,9 \%$ \\
\hline Acerto geral & $58,9 \%$ & $25,5 \%$ & $7,1 \%$ & $8,5 \%$ & $80,1 \%$ \\
\hline
\end{tabular}

Fonte: Dados da pesquisa.

Verifica-se que o modelo gerado apresenta um poder preditivo de 80,1\% (ACC global). Cabe salientar que em relação ao pior risco (D) e o melhor risco (A), o modelo apresenta um poder preditivo de $90,9 \%$ e $89,9 \%$ respectivamente.

Por fim, para avaliar a capacidade de generalização do modelo, o mesmo foi ajustado à base de teste para execução de uma validação cruzada. A validação cruzada consiste em aplicar o modelo gerado pela subamostra de estimação tendo como base uma subamostra de validação e verificar o resultado do poder preditivo para essa nova subamostra. Os resultados são apresentados na Tabela 8.

Tabela 8 - Poder preditivo na subamostra de validação

\begin{tabular}{c|r|r|r|r|r}
\hline \multirow{2}{*}{ Observado } & \multicolumn{7}{c}{ Classificação Prevista } \\
\cline { 2 - 6 } & A & B & C & D & Percentual de acerto \\
\hline A & 46 & 10 & 0 & 0 & $82,1 \%$ \\
B & 3 & 12 & 0 & 0 & $80 \%$ \\
C & 0 & 0 & 0 & 0 & $0 \%$ \\
D & 0 & 0 & 0 & 0 & $0 \%$ \\
\hline Acerto geral & $69 \%$ & $31 \%$ & $0 \%$ & $0 \%$ & $81,7 \%$ \\
\hline
\end{tabular}

Fonte: Dados da pesquisa.

Para o conjunto de teste, no qual se observou apenas classificações à priori nas classes de risco $\mathrm{A}$ e $\mathrm{B}$, os resultados apresentaram um $\mathrm{ACC}$ geral de $81,7 \%$, superior ainda à classificação do modelo geral.

\section{CONCLUSÃO}

O presente estudo teve como objetivo estimar um modelo de classificação (rating) de Cooperativas de Crédito de acordo com o risco de crédito que elas representam para outros agentes no mercado financeiro. As variáveis explicativas do rating foram definidas com base 
em indicadores do modelo PEARLS. Os dados são referentes a Cooperativas de Crédito singulares que tiveram suas contas analisadas por um grande banco de varejo nacional e foram coletados junto ao sistema de informações do BACEN.

O modelo multinomial estimado foi baseado em um conjunto de 23 (vinte e três) indicadores do modelo PEARLS e no índice de Basiléia disponível no sistema do BACEN. Após análise do conjunto de dados e reclassificação da variável de resposta em quatro classes de rating, a pesquisa procedeu uma análise de componentes principais para garantir ortogonalidade entre as variáveis explicativas e consequente ausência de multicolinearidade. Para a estimação do modelo foi aplicada a técnica stepwise com o critério Wald em um conjunto de dados de treinamento (amostra de treinamento) para que as variáveis selecionadas fossem estatisticamente significativas.

Desse processo resultou um modelo estatisticamente significativo com Nagelkerke Pseudo- $\mathrm{R}^{2}$ de 0,783 com apenas cinco variáveis que cobriram cinco dos seis pilares do modelo PEARLS. Este modelo, apesar de sua baixa complexidade, foi capaz de gerar uma acurácia de $80,1 \%$ na amostra de treinamento e $81,7 \%$ na amostra de teste.

Com os resultados obtidos pelo modelo estimado, sobretudo devido ao seu grau de acurácia, é possível verificar que o uso do modelo PEARLS, juntamente com as técnicas estatísticas empregadas, permitiu o desenvolvimento de um modelo para monitoramento do risco que as cooperativas de crédito representam para o mercado financeiro. O modelo de rating desenvolvido se mostrou simples e estatisticamente significativo.

Como sugestão para trabalhos futuros, recomenda-se ampliar a amostra e fazer uma análise considerando grupos distintos de cooperativas, como as mais voltadas para crédito rural, para o público urbano e para a economia solidária e microcrédito.

\section{REFERÊNCIAS}

BACEN, Banco Central do Brasil. Panorama do sistema nacional de crédito cooperativo. Recuperado de: https://www.bcb.gov.br/content/estabilidadefinanceira/ coopcredpanorama/panorama_de_cooperativas2017.pdf. Acesso em 03/11/2018.

BALIOS, D.; THOMADAKIS, S.; TSIPOURI, L. Credit rating model development: an ordered analysis based on accounting data. Research in International Business and Finance, v. 38, p. 122-136, 2016.

BARROSO, M. F. G.; BIALOSKORSKI NETO, S. Análise do spread da intermediação financeira em cooperativas de crédito. Contabilidade Vista \& Revista, v. 23, n. 3, p. 145$171,2012$.

BIALOSKORSKI NETO, S. Economia e gestão de organizações cooperativas. 2. ed. São Paulo: Atlas, 2012.

BRESSAN, V. G. F.; BRAGA, M. J.; BRESSAN, A. A.; RESENDE FILHO, M. A. Uma proposta de indicadores contábeis aplicados às cooperativas de crédito. Revista de Contabilidade e Controladoria, v. 2, n. 4, p. 58-80, 2010.

BRESSAN, V. G. F., BRAGA, M. J., BRESSAN, A. A. \& RESENDE FILHO, M. A. Avaliação de insolvência em cooperativas de crédito: uma aplicação do sistema PEARLS. Rev. Adm. Mackenzie, v. 12, n. 2, p. 113-144, 2011.

CARREIRO, L. C.; CUNHA, M. A. Análise do desempenho econômico-financeiro do Banco Cooperativo do Brasil SA - BANCOOB pela metodologia CAMEL. In: Congresso Brasileiro de Custos, XV, 2008. Anais... ABC, nov.

CREPALDI, J. A. L.; PAYÉS, M. A. M. O papel dos bancos públicos na crise financeira 
Rating para Avaliação de Cooperativas de Crédito: Uma Aplicação do Modelo PEARLS

internacional de 2008. €CO\$: Revista de Estudos em Economia, v. 4, p. 1-74, 2015. FÁVERO, L. P. L.; BELFIORE, P. P. Manual de análise de dados: estatística e modelagem multivariada com excel, SPSS e STATA. Rio de Janeiro: Elsevier, 2017.

FGCOOP. Fundo Garantidor do Cooperativismo de Crédito, 2017. Recuperado de: https:// www.fgcoop.coop.br. Acesso em 11/04/2018.

GUEVARA, C. A.; POLANCO, D. Correcting for endogeneity due to omitted attributes in discrete-choice models: The multiple indicator solution. Transportmetrica A: Transport Science, v. 12, n. 5, p. 458-478, 2016. http://dx.doi.org/10.1080/23249935.2016.1147504

HAIR Jr, J. F.; BLACK, W. C.; BABIN, B. J.; ANDERSON, R. E.; TATHAM, R. L. Análise Multivariada de Dados, 6 ed., Porto Alegre: Bookman, 2009.

LIMA, F. G.; FONSECA, C. V. C.; SILVEIRA, R. L. F.; ASSAF NETO, A. Os Determinantes dos Ratings de Crédito dos Bancos Brasileiros. Revista de Administração Contemporânea, v. 22, n. 2, p. 178-200, 2018.

MEINEN, E. Cooperativismo financeiro: percurso histórico, perspectivas e desafios. Brasília: Confebras, 2014.

NAKAMURA, L. I.; ROSZBACH, K. Credit ratings, private information, and bank monitoring ability. Journal of Financial Intermediation, v. 36, October, p. 58-73, 2018.

RICHARDSON, D. C. PEARLS Monitoring System. World Council of Credit Unions. Toolkit series number 4. April, 2009. Recuperado de: https://www.woccu.org/documents/ pearls_monograph. Acesso em 03/04/2019.

SILVA, J. P. Gestão e Análise de Risco de Crédito. 7 ed. São Paulo: Atlas, 2013.

SILVEIRA, R. A. C.; TAKAMATSU, R. T.; AVELINO, B. C. Impacto dos Ratings de Crédito nas Ações de Empresas de Capital Aberto no Brasil. RACE: Revista de Administração, Contabilidade e Economia, v. 16, n. 2, p. 573-602, 2017.

VASCONCELOS, R. W. B. Identificação de indicadores econômico-financeiros para análise de cooperativas de crédito, singulares ou centrais. Departamento de Supervisão Indireta e Gestão da Informação (DESIG), Banco Central do Brasil. Belo Horizonte: Banco Central do Brasil, 2006. 